\title{
COMPARISON OF TURBULENCE IN HII REGIONS AND MOLECULAR CLOUDS
}

\author{
C. R. O'DELL \\ Department of Space Physics and Astronomy \\ Rice University \\ P. O. Box 1892 \\ Houston, Texas, 77251 USA
}

\begin{abstract}
Both the HII Regions and the Molecular Clouds show broadening of their emission lines beyond that expected from thermal motion and this is ascribed to turbulence. Turbulence in molecular clouds generally agrees with a model where the velocity of motion is determined by the Alfvén velocity.

Turbulence in Galactic HII Regions and Giant Extragalactic HII Regions can also be studied by the width of the emission lines. The magnitude of the turbulent velocities in these regions are characteristically about $10 \mathrm{~km} / \mathrm{s}$. There is a general increase in turbulent velocity with the size of the HII Region, and this relation is close to but different from the one third power dependence expected from the most naive application of Kolmogorov theory. When a detailed study is conducted of each Galactic HII Region by means of the structure function, one finds that there is not agreement with Kolmogorov theory.

The Size-Turbulent versus Velocity relation for Galactic HII Regions differs slightly from the better defined velocity relation for Giant Extragalactic HII Regions. This difference is probably due to the fact that the larger extragalactic objects are probably complexes of multiple individual HII Regions. There is no evidence that broadening of extragalactic HII Regions is due to motion about a common center of mass.
\end{abstract}

\section{TURBULENCE IN MOLECULAR CLOUDS}

Broadening of radio molecular emission lines arising from these cold regions generally is greater than expected from thermal broadening. This extra broadening is small, but certainly real, in the Cores of these clouds (e.g., Meyers 1983) and is very pronounced in studies of the full Molecular Complexes (e.g., Dame et al. 1986). The results of these studies are shown in Figure 1, where the data is grouped by intervals in the logarithm in the diameters.

There is a rudimentary theory that can explain the increase in turbulent velocity with size that is seen in the Molecular Clouds. As shown in the reviews by Meyers (1987) and Falgarone and Perault (1987) there is a general agreement with the one half power law dependence that would apply if the turbulent velocities were limited by the Alfven wave velocities. We show in the figure that the slope of the 
relation determined from the cores and the complexes is very similar and taken together has a slope of $\mathbf{0 . 5 2}$, which is remarkably close to the value of one half expected from the magnetic model.

\section{LINE BROADENING IN EXTRAGALACTIC HII REGIONS}

The existence of a general relationship between line width and size of Giant Extragalactic HII Regions (GEHR) was first suggested by Melnick (1977), who argued that the large velocities were due to large scale kinematic motion about a center of mass. This interpretation did not recognize that similarly large line widths were already seen in the Galactic HII Regions. Roy et al. (1986) have determined the size-velocity data set with the greatest accuracy and the figure also shows their grouped results. These data are corrected for thermal broadening.

\section{LINE BROADENING IN GALACTIC HII REGIONS}

In many ways it is more difficult to get a measure of the total turbulent velocity in the Galactic HII Regions (GHIIR) because of their large angular sizes. My colleagues and I have been mapping velocities across the faces of GHIIR for over a decade in order to accurately model the evolution of these objects (c.f. references in O'Dell and Castañeda 1987). In a given line of sight we see a particular value of the line width and we also see random variations in the radial velocity from point to point across the face of the nebulae. The dispersion of values of the radial velocities can be used to correct the average observed line width $\beta$ through the equation

$$
\beta=\left(\beta_{o b s}{ }^{2}+2 \times S D^{2}\right)^{1 / 2}
$$

where SD is the standard deviation. Figure 1 also shows the results for the turbulence in GHIIRs, with the values representing the entire nebulae in the same fashion as the GEHRs and the Molecular Clouds.

Examination of the figure shows that one can fit a simple curve of the form log $\beta=0.78+.25 \log \mathrm{D}$ to all of the HII region data, both Galactic and extragalactic. This slope is close but demonstrably different from the expectation of $1 / 3$ that would result if all HII regions were similar and naively behaved according to Kolmogorov theory for an incompressible gas. Although this theory has many inapplicable assumptions, it agrees with laboratory and measurements made in nature for a wide variety of gases and fluids. As such, it is not surprising that there would be general agreement in this astronomical application of the theory.

The data indicate that there may be a slightly different relation of size and velocity for the GHIIR and the GEHR. This would not be unexpected, as the GHIIR are clearly single objects while the GEHR are probably multiple HII Regions that are not resolved spatially because of their great distances. 


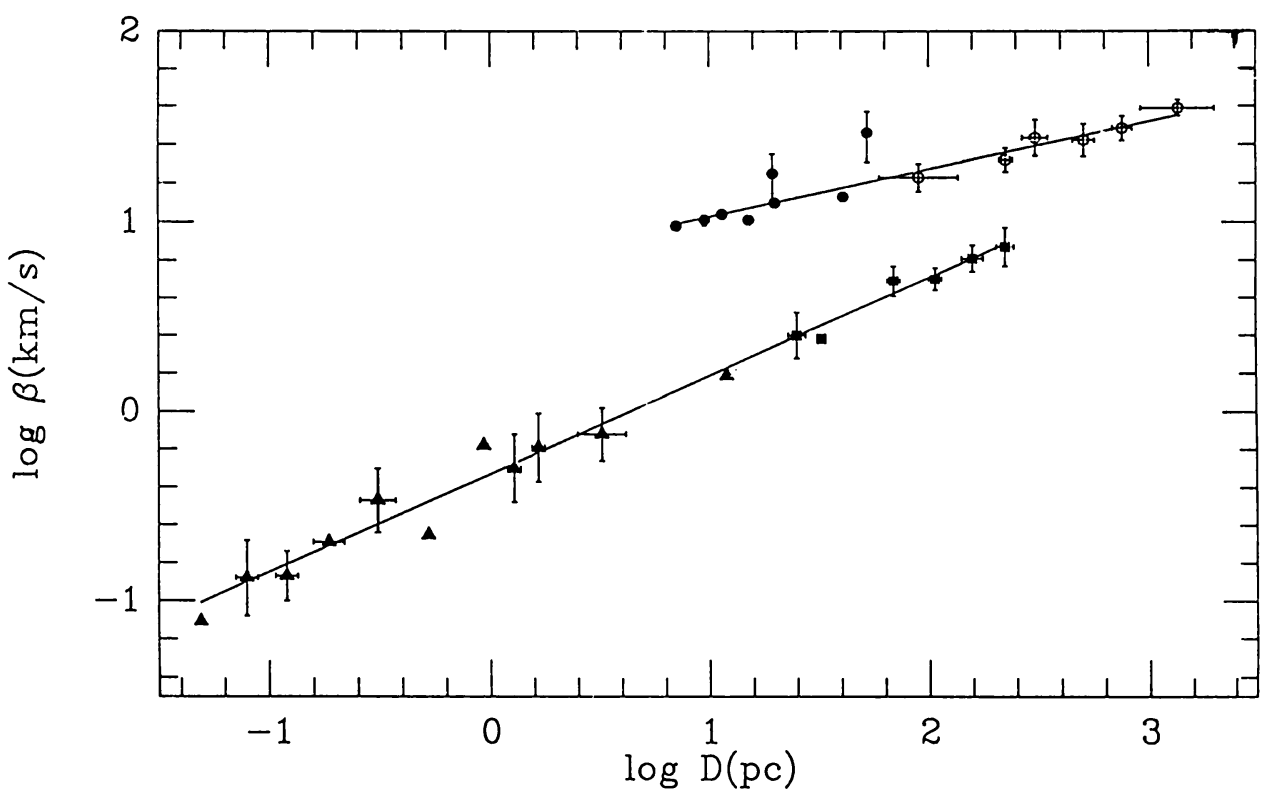

Figure 1. The relation between size and $e^{-1}$ velocity width is shown for the Cores $(\boldsymbol{\Delta})$, Complexes ( $\boldsymbol{a})$, GEHRs (0), and GHIIRs ( $\bullet$ ). The solid lines are the corresponding least square fits for each kind of object. The Core's data are for the turbulent component of motion.

\section{DETAILED TESTS OF KOLMOGOROV THEORY IN GALACTIC HII REGIONS.}

It is possible to use the detailed study of random radial velocity variations across the face of the GHIIR's to test the applicability of Kolmogorov theory. The theoretical framework for this was established by von Hoerner (1951) who showed that the most useful statistical measure of the random velocities is the structure function

$$
B(r)=\left\langle\left|V\left(r^{\prime}\right)-V\left(r^{\prime \prime}\right)\right|^{2}\right\rangle
$$

This function should have a slope of $5 / 3$ in the inner region of a nebula, begin to flatten when the average separation is comparable to the depth of the nebula, and would be $2 / 3$ if one ever reached distances much larger than the depth of the nebula. The results from the study of a number of GHIIR (O'Dell and Castañeda 1987) demonstrates that when studied in detail the Galactic HII Regions do not agree with Kolgomorov theory. This means that general relationship between size and turbulent velocity inferred from the line broadening must reflect some mechanism different from Kolgomorov

theory. 


\section{CONCLUSIONS}

Our summary of the combined data for cores and entire molecular cloud complexes shows that the theory of random motions of material being limited by the Alfven wave velocities can explain the observed line broadening over a size range of more than three orders of magnitude.

The internal velocities in Galactic HII Regions and Giant Extragalactic HII Regions shows a well defined correlation with size over a size range of a factor over two orders of magnitude but with a very different slope than that for molecular clouds. The continuity between Galactic and extragalactic objects argues against the latter being broadened by large scale kinematic motions.

Detailed determination of the turbulent velocities within Galactic HII Regions from studies of the radial velocities along various lines-of-sight indicates that there is not any agreement between the observations and the simplest application of Kolmogorov theory to these nebulae.

\section{REFERENCES}

Dame, T. M., Elmegreen, B. G., Cohen, R. S., and Thaddeus, P. (1986) Ap.J., $305,892$.

Falgarone, E. and M. Perault (1987) in Physical Processes in Interstellar Clouds, ed. G.E. Morfill and M. Scholer (Dordrecht: Reidel) p. 59.

Melnick, J. (1977) Ap. J., 213, 15.

Meyers, P. C. (1983) Ap. J., 270, 105.

Meyers, P. C. (1987) in Interstellar Processes, ed. D. J. Hollenbach and H. A Thronson, Jr. (Dordrecht: Heidel) p. 71.

O'Dell, C. R. and Castañeda, H. O. (1987) Ap. J., 317, 686.

Roy, J. R., Arsenault, R. and Joncas, G. (1986) Ap.J., 300; 626.

von Hoerner, S. (1951) Zs.Ap., 30, 17. 